\title{
Mieloma Múltiplo: Uma nova ótica sob a abordagem diagnóstica- Relato de caso
}

\author{
Multiple myeloma: A new optic under the diagnostic approach- case report
}

Thiago Wesley de Oliveira ${ }^{\dagger *}$, Paulo Sérgio Lopes Soares ${ }^{\dagger}$, Tiago de Oliveira Boechat

Como citar esse artigo. de Oliveira,

TW; Soares, PSL; Boechat, TO. Mieloma Múltiplo: Uma nova ótica sob a abordagem diagnóstica- Relato de caso. Revista de Saúde. 2018 Jul./Dez.; 09 (2): 37-43

\begin{abstract}
Resumo
O mieloma múltiplo é um tumor maligno caracterizado por proliferação de plasmócitos na medula. O tema atinge maior relevância pelo aumento na expectativa de vida da população, já que acomete mais idosos. Este trabalho objetivou relatar um caso de mieloma e sua abordagem no estágio tardio. Indivíduo do sexo masculino, 85 anos, com queixas de astenia, oligúria e dor lombar há 3 meses, foi admitido no Hospital Universitário de Vassouras (HUV) e chamava a atenção pela intensa palidez cutâneo- mucosa. Ressonância magnética revelou lesões ósseas líticas na região sacral e mielograma exibiu plasmocitose medular compatível com mieloma múltiplo, porém logo após o diagnóstico o paciente evoluiu para óbito por sepse nosocomial. Bibliografia ressalta a importância do reconhecimento precoce para a maior sobrevida a partir do tratamento instituído. Portanto, mesmo sendo uma doença incurável, o diagnóstico antecipado favorece tratamento precoce, melhoria da qualidade de vida e maior sobrevida para esses pacientes.
\end{abstract}

Palavras-chave: Diagnóstico precoce; Mieloma Múltiplo; Atenção Primária à Saúde.

\begin{abstract}
Multiple myeloma is a malignant tumor characterized by the proliferation of plasma cells in the bone marrow. The issue has currently become more relevant also due to the increase of life expectancy of the population, since it affects more elderly people. This work aims to report a case of myeloma in advanced stages and our approach to it. A 85-year-old man, admitted to Hospital Universitário de Vassouras (HUV), who had complaints about asthenia, oliguria and low back pain was noticed for intense mucosal-skin paleness. Abdominal magnetic resonance imaging showed lytic bone lesions in the sacral region and the myelogram presented medullary plasmacytosis compatible with multiple myeloma. However, soon after the diagnosis, the patient progressed to death due to nosocomial sepsis. Bibliography emphasize the importance of early detection to the success rate of the treatment. Therefore, even though the disease is incurable, the earlier the patient gets diagnosed, the more effective treatment is in improving the quality and longevity of life.

Keywords: Early Diagnosis; Multiple Myeloma; Primary Health Care.
\end{abstract}

\section{Introdução}

O Mieloma Múltiplo (MM) é uma neoplasia hematológica que, uma vez diagnosticada precocemente, pode proporcionar melhores perspectivas de vida para os pacientes. Esse assunto tem atingido maior relevância atualmente não só pelo seu impacto sobre a saúde pública mas pelo aumento na expectativa de vida da população, já que acomete mais indivíduos adultos e idosos $^{1}$.

O MM representa um tumor progressivo e incurável de células $\mathrm{B}$, definido pela multiplicação desregulada e clonal de plasmócitos com produção de imunoglobulina (Ig) monoclonal denominada proteína $\mathrm{M}^{2}$. É a segunda neoplasia hematológica mais comum. Ocorre geralmente em pessoas com idade em torno de 65 anos, com maior incidência em homens ${ }^{2}$.

As consequências clínicas do MM são o comprometimento de órgãos alvo, especialmente a anemia, dor óssea, insuficiência renal e hipercalcemia (reconhecidas como CRAB) ${ }^{3}$. A anemia é a apresentação clínica mais comum e por vezes a única a se manifestar no início da doença. A não investigação correta da anemia, especialmente acima dos 60 anos de idade pode fazer com que o MM seja subdiagnosticado ${ }^{4}$. Sem sinais peculiares, a conclusão diagnóstica desse tumor pode ser tardia, momento em que já estão instaladas sobreposição de complicações, limitando, assim, a possibilidade terapêutica ${ }^{5}$.

Os critérios diagnósticos foram atualizados em $2016^{6}$ e estão demonstrados no Quadro 1.

Afiliação dos autores: $\uparrow$ Universidade de Vassouras, Pró-Reitoria de Ciências Médicas, Discente do curso de Medicina;

\$ Universidade de Vassouras, Pró-Reitoria de Ciências Médicas, Docente do curso de Medicina

*twoliveira1987@hotmail.com 
Do ponto de vista da avaliação complementar, a eletroforese de proteínas no soro/urina, a imunofixação e dosagem de cadeias leves livres (FreeLight) são os métodos de eleição para a detecção dos picos monoclonais $\mathrm{M}^{7}$. Os dois últimos são mais sensíveis para identificar baixas concentrações do componente M nos casos de pacientes com MM de cadeia leve e oligosecretor: pacientes que secretam pequenas quantidades de componente $\mathrm{M}$ no soro, que representa até um terço dos pacientes acometidos por essa patologia ${ }^{1}$.

Além disso, é necessária a análise da medula óssea através do mielograma e biópsia, que revelam a quantidade de plasmócitos na medula óssea ${ }^{8}$.Já para avaliação das lesões osteolíticas, os exame mais utilizados são radiografia, tomografia e ressonância magnética nuclear (RM) ${ }^{7}$.

A causa do MM é desconhecida, porém pode ser influente a exposição a benzeno, radiações e inseticidas? ${ }^{7}$.

Este trabalho objetivou relatar um caso de mieloma e alertar os médicos para o reconhecimento da doença em um estágio inicial de evolução, proporcionando assim, tratamento precoce e maior sobrevida.

\section{Descrição do caso}

Um paciente do gênero masculino, 85 anos, negro, aposentado, natural de Seropédica- RJ, queixouse de astenia, perda ponderal, intolerância aos médios esforços, oligúria e dor lombar de início insidioso, em pontada, associada ao movimento. Procurou a Unidade Básica de Saúde (UBS) após 3 meses com esses sintomas, sendo prescrito analgésico e sulfato ferroso devido anemia detectada no hemograma. Sem melhora do quadro, dez dias depois foi admitido no Hospital Universitário de Vassouras (HUV) deambulando com auxílio de guia, em regular estado geral, com clínica de constipação intestinal, oligúria, cansaço evidente e dor lombar. Informou piora da sensação de fraqueza e inapetência nas últimas horas. Alegava hipertensão controlada de longa data e hiperplasia prostática benigna (HPB), sem outras comorbidades. Ao exame físico chamava atenção uma intensa palidez cutâneomucosa, edema frio em membros inferiores $(3+/ 4+)$ com sinal de cacifo e sensibilidade normal; abdome hipertimpânico com macicez supra- púbica. Exames demonstraram: anemia (hemoglobina 9,5 mg/dl), VCM $96 \mathrm{fL}, \mathrm{CHCM} 35 \mathrm{~g} / \mathrm{dL}$, hipercalcemia $(1,59 \mathrm{mmol} / \mathrm{L})$, creatinina de $11 \mathrm{mg} / \mathrm{dl}$, ureia $245 \mathrm{mg} / \mathrm{dl}$, sorologia não reagentes para hepatite $\mathrm{B}$, hepatite $\mathrm{C}, \mathrm{HIV}$. À tomografia de pelve, solicitada para investigar a dor lombar, foram visualizadas lesões ósseas líticas comprometendo corpos vertebrais ao nível do sacro com formação de massas com densidade de partes moles (Figura 1). A condução do caso consistiu, a princípio, em estabilização clínica, sondagem vesical de demora, dieta laxativa, hemodiálise e controle sintomático. Simultaneamente, realizou- se a procura pelo foco neoplásico. $\mathrm{O}$ exame urológico não revelou indícios de câncer, apenas HPB. No entanto, no transcurso da internação, permaneceu restrito ao leito, evoluiu progressivamente com paraplegia de membros inferiores, anorexia gradativa e piora da dor. Foi solicitado estudo tomográfico do crânio, por meio da qual verificou-se múltiplas lesões osteolíticas na calota craniana (Figura 2). Diante dessas evidências e da persistência de síndrome consumptiva, anemia e queda do estado geral, foi então priorizada avaliação hematológica a fim de se elucidar critérios que corroborassem com a suspeição de MM. A eletroforese de proteínas exibiu uma fração pequena de componente monoclonal em gamaglobulinas $(0,1 \mathrm{mg} / \mathrm{dl})$, como pode ser visto na Figura 3. O mielograma evidenciou 35\% de plasmoblastos, confirmando a hipótese diagnóstica de MM (Figura 4). Concomitante a essa investigação, o paciente evoluiu com febre, torpor e infecção pelo cateter. Foi então iniciado esquema antimicrobiano com gentamicina $80 \mathrm{mg}$ e vancomicina $1 \mathrm{~g}$ por cinco dias. Para o tratamento do MM foi iniciado dexametasona $20 \mathrm{mg} /$ dia durante 4 dias intercalado com 4 dias sem corticoide. Dois dias após confirmação diagnóstica, o paciente evoluiu para óbito devido complicações da sepse nosocomial.

\section{Discussão}

O MM é uma doença heterogênea e calcula-se que a sobrevida do paciente com MM seja de meses a vários $\operatorname{anos}^{2}$. A incidência do mieloma é maior em pessoas idosas e a sobrevida depende muito da fase em que se descobre a doença ${ }^{3}$.

Considerando a realidade demográfica, sabe- se que o Brasil passa por um processo de envelhecimento populacional acelerado. A temática sobre $\mathrm{MM}$ vem assumindo expressiva importância com a melhora na expectativa de vida, pois aumentou a quantidade de pessoas vivendo na faixa etária de maior incidência dessa doença, gerando maior interesse na avaliação dessa população específica9 ${ }^{9}$. Como consequência, espera-se, nos próximos anos, um aumento progressivo na procura dos serviços de saúde por pacientes com queixas inespecíficas de anemia e dores no corpo, exigindo um olhar crítico do médico para se atentar para MM como diagnóstico diferencial nesse quadro ${ }^{5}$.

Sob o aspecto propedêutico, o fundamento do diagnóstico do MM é a presença do componente monoclonal no soro ou urina aliada à identificação de plasmocitosemedular em um paciente que apresente anemia isolada ou, especialmente, quando associada à queixas de dores ósseas e/ou evidências de hipercalcemia 
e insuficiência renal. Esse contexto clínico num paciente acima de 60 anos torna ainda mais provável o diagnóstico ${ }^{6}$.

Tendo em vista que o paciente desse relato, idoso, apresentou- se com dores lombares, anemiado e insuficiência renal, valorizou- se a suspeição de MM. O direcionamento da investigação diagnóstica corrobora com o preconizado na literatura: realização de eletroforese e mielograma/BMO (Biópsia de Medula Óssea), que revelaram pico monoclonal e plasmocitose de $35 \%{ }^{8}$.

Considerando a queixa principal do paciente retratado, a lombalgia compreende uma apresentação básica dessa patologia. Dois terços dos pacientes têm dor óssea nas costas relacionada ao movimento ${ }^{10}$, uma vez que a hiperplasia plasmocitária leva à hiperfunção dos osteoclastos, resultando em lesões osteolíticas ${ }^{11}$.

Uma alteração esperada na evolução natural dessa doença debilitante é a paresia e paraplegia dos membros inferiores, verificada nessa história descrita. Nesse sentido, segundo Marta, Falcão e Saavedra ${ }^{12}$, complicações neurológicas, como a radiculopatia lombo- sacra, resultam da compressão do nervo pela lesão vertebral osteolítica na fase avançada do $\mathrm{MM}^{12}$. Logo, pode-se inferir que o paciente apresentava-se em doença com estágio avançado porque apresentava manifestações do $\mathrm{CRAB}$ e complicação neurológica.

Outro aspecto interessante é o fato de que, simultâneo ao momento em que se fechou o diagnóstico de MM, o paciente encontrava- se grave, séptico e comatoso. Ou seja, evoluiu com disfunções orgânicas em um contexto de infecção. A progressão clínica observada nesse caso comprova o que foi revelado em outros trabalhos: complicações infecciosas constituem a mais importante causa de óbito de $\mathrm{MM}^{6}$. Essa condição acontece sobretudo em razão da imunodeficiência associada à doença e do tratamento implementado no paciente ${ }^{3}$.

Dentre os diagnósticos diferenciais possíveis para o caso de um idoso com anemia, hipercalcemia, insuficiência renal e lesões líticas, deve-se pensar especialmente em outra doença neoplásica commetástase óssea e a biópsia de medula óssea é fundamental para essa distinção?

Tendo em vista o tratamento antitumoral, ele deve ser realizado exclusivamente em doentes com MM sintomático e pode prolongar a qualidade de vida e a sobrevida ${ }^{9}$. É realizada terapia para controle da doença e tratamento de suporte para prevenir morbidade.

As opções são muitas: radioterapia, quimioterapia, terapias de suporte ou transplante autólogo de medula óssea. Mas a quimioterapia é o tratamento de eleição nos doentes sintomáticos ${ }^{13}$.

Pacientes que não são candidatos à transplante devido à idade e comorbidades, realizam protocolos quimioterápicos que idealmente associam duas ou três drogas como corticóide, agentes imunomoduladores, drogas alquilantes e inibidores de proteassoma. O corticóide é uma droga eficaz no tratamento e no alívio dos sintomas, disponível em todos os hospitais, por isso, em centros que não dispõem de tratamento oncohematológico, pode ser realizado monoterapia inicial com corticóide, como foi feito no caso descrito. Mas a decisão de efetuar terapêutica depende do quadro clínico-laboratorial do doente, estando indicada em pacientes sintomáticos. ${ }^{9}$

No caso descrito, após o diagnóstico, foi iniciado bloco de dexametasona em pulso como preconizado pela literatura ${ }^{13}$. A dose foi reduzida $(20 \mathrm{mg} /$ dia $)$ devido a idade e o quadro séptico vigente.

Antes da internação o paciente já havia procurado atendimento na UBS com queixas e alterações laboratoriais que poderiam sugerir a investigação de MM. Essa realidade prova que MM se desenvolve de forma silenciosa com quadro sindrômico inespecífico ${ }^{6}$, obrigando o médico a considerar MM como diagnóstico diferencial, sobretudo quando trata-se de idoso.

Nesse trabalho relatado, o quadro anêmico se instalou de modo insidioso e progrediu na medida da progressão da doença de base. A Atenção Primária identificou essa condição, mas a investigação etiológica não foi valorizada naquele momento. A postura da equipe da UBS foi tratar empiricamente a anemia com sulfato ferroso, não havendo ponderado sobre possíveis diagnósticos diferenciais frente a um paciente idoso com anemia, dor lombar e oligúria ${ }^{2}$.

O raciocínio clínico dos profissionais da UBS deve ser mais crítico em casos de idosos com dor óssea e anemia, ${ }^{14}$ pois somente quando o paciente buscou o nível de Atenção Secundária é que se atentou para diagnósticos diferenciais. Exames relevantes que podem ser realizados na atenção primária para essa suspeita são, além do hemograma e bioquímica, eletroforese de proteínas e inventário ósseo por radiografia simples. Um fluxograma proposto por Silva et al. ${ }^{5}$ (Figura 5) resume bem a orientação que o profissional da atenção básica deve seguir frente a suspeita clínica de MM.

AssimoencaminhamentoparaAtençãoSecundária poderá ser antecipado, permitindo o diagnóstico mais precoce $^{15}$. Além disso, a assistência especializada passa a receber esses pacientes em condições de tratamento com alvo no binômio sobrevida-qualidade de vida ${ }^{16}$.

Em termos de gerenciamento, o manejo do paciente com MM em estágio avançado, como o contexto desse relato, é mais caro do que em estágio inicial para o SUS. Essa doença quando detectada tardiamente relaciona-se com maior número de complicaçõese assim mais internações (prolongadas), mais sessões de hemodiálise, antibioticoterapia e necessidade de reabilitação neurológica por exemplo ${ }^{16}$.

Portanto, é relevante que os médicos valorizem sinais inespecíficos como a anemia, principalmente em 
Quadro 1. Critério Internacional sobre Diagnóstico de Mieloma Múltiplo de acordo com Rajkumar'.

Ambos os critérios devem ser atendidos:
Células plasmáticas clonais de medula óssea $\geq 10 \%$ ou plasmocitoma ósseo ou extramedular comprovado por biópsia

\begin{abstract}
Qualquer um ou mais dos seguintes eventos definidores de mieloma:

Evidência de lesão de órgão terminal que pode ser atribuída ao distúrbio proliferativo de células plasmáticas subjacente, especificamente
\end{abstract}

Hipercalcemia: cálcio sérico $>0,25 \mathrm{mmol} / \mathrm{L}(>1 \mathrm{mg} / \mathrm{dL})$ acima do limite superior do normal ou $>2,75 \mathrm{mmol} / \mathrm{L}(>11 \mathrm{mg} / \mathrm{dL})$

Insuficiência renal: clearance de creatinina $<40 \mathrm{~mL} / \min$ ou creatinina sérica $>177 \mu \mathrm{mmol} / \mathrm{L}(>2 \mathrm{mg} / \mathrm{dL})$

Anemia: valor de hemoglobina de $>2 \mathrm{~g} / \mathrm{dL}$ abaixo do limite inferior do normal, ou um valor de hemoglobina $<10 \mathrm{~g} / \mathrm{dL}$

Lesões ósseas: uma ou mais lesões osteolíticas na radiografia do esqueleto, tomografia computadorizada (TC) ou PET-CT

Porcentagem clonal de plasmócitos na medula óssea $\geq 60 \%$

O nível de FLC envolvido deve ser de

$\geq 100 \mathrm{mg} / \mathrm{L}$

$>1$ lesão focal em estudos de ressonância magnética (pelo menos 5 mm de tamanho)

Figura 1. Lesão lítica no sacro com formação de massas (seta) com densidade de partes moles

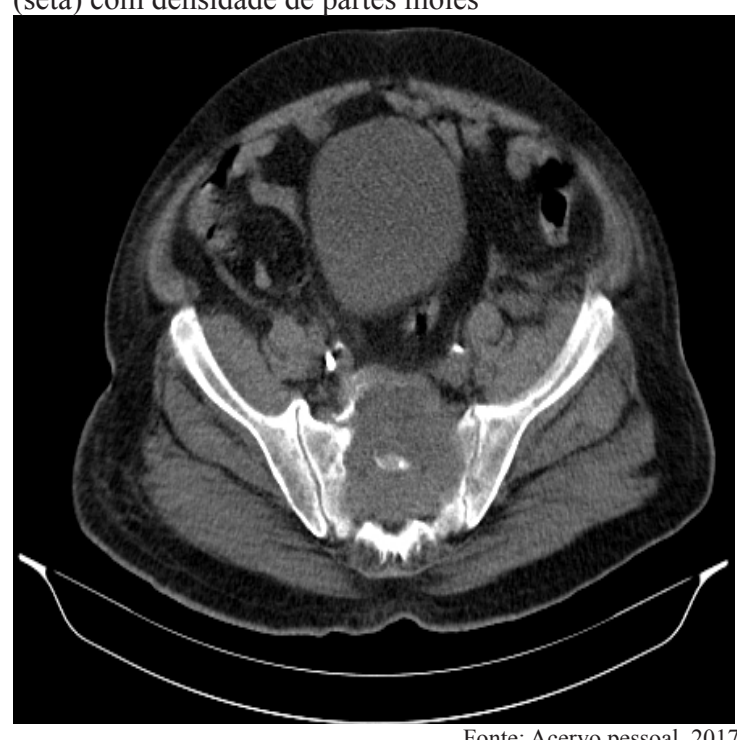

Fonte: Acervo pessoal, 2017 


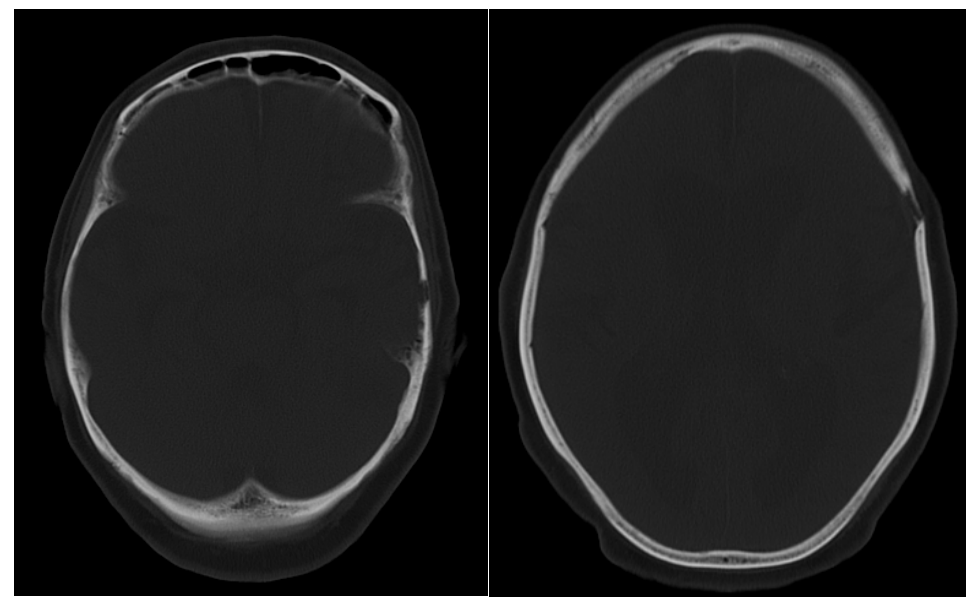

Figura 2. Pequenas áreas de lesões líticas (setas) na janela óssea.

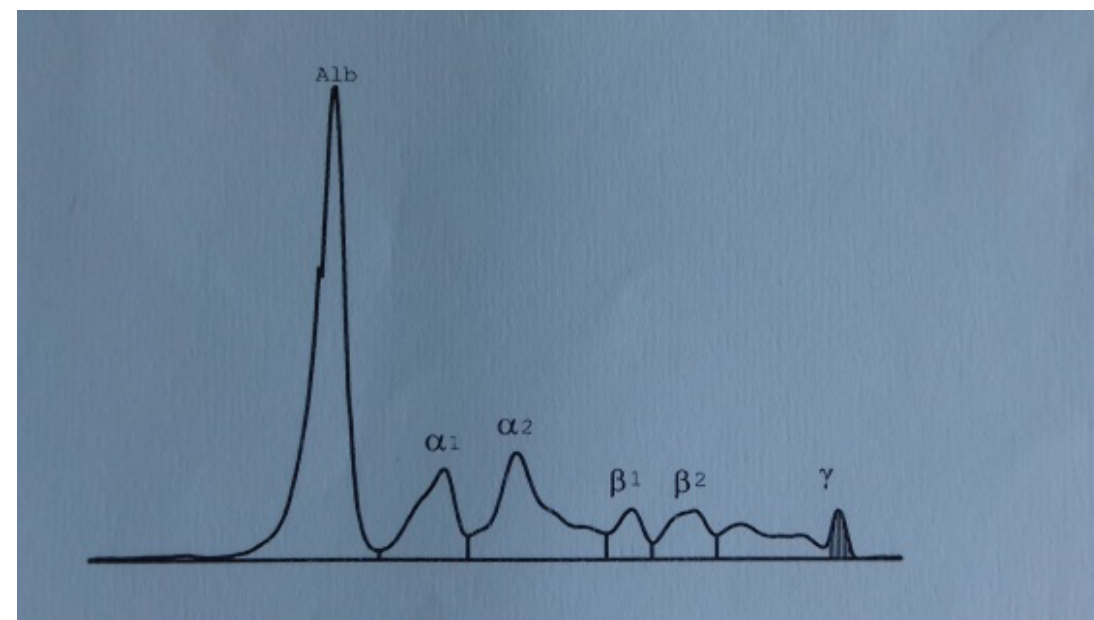

Figura 3. Gráfico exibe uma pequena fração de componente monoclonal em gamaglobulinas $(0,1 \mathrm{mg} / \mathrm{dl})$.

Figura 4. Mielograma exibindo plasmocitose com plasmócitos multinucleados (seta preta) e com vacúolos (seta branca)contendo imunoglobulinas monoclo

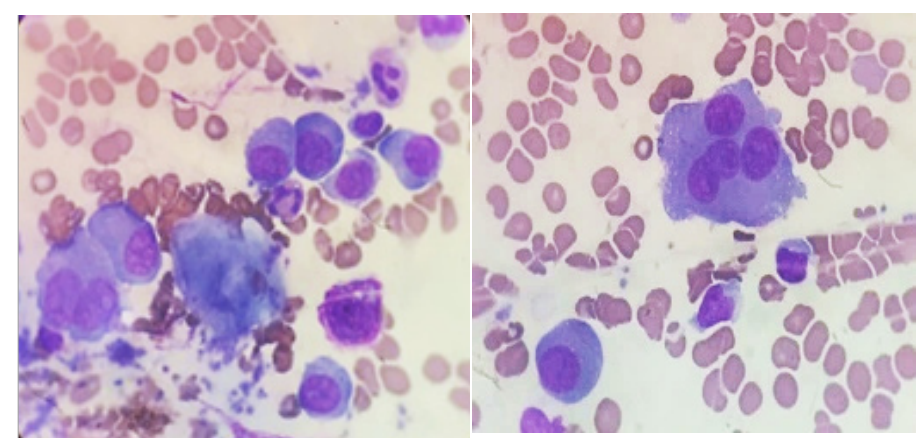


Figura 5. Fluxograma de investigação do paciente com suspeita de MM. (Fonte: Adaptado de Silva et al.5).

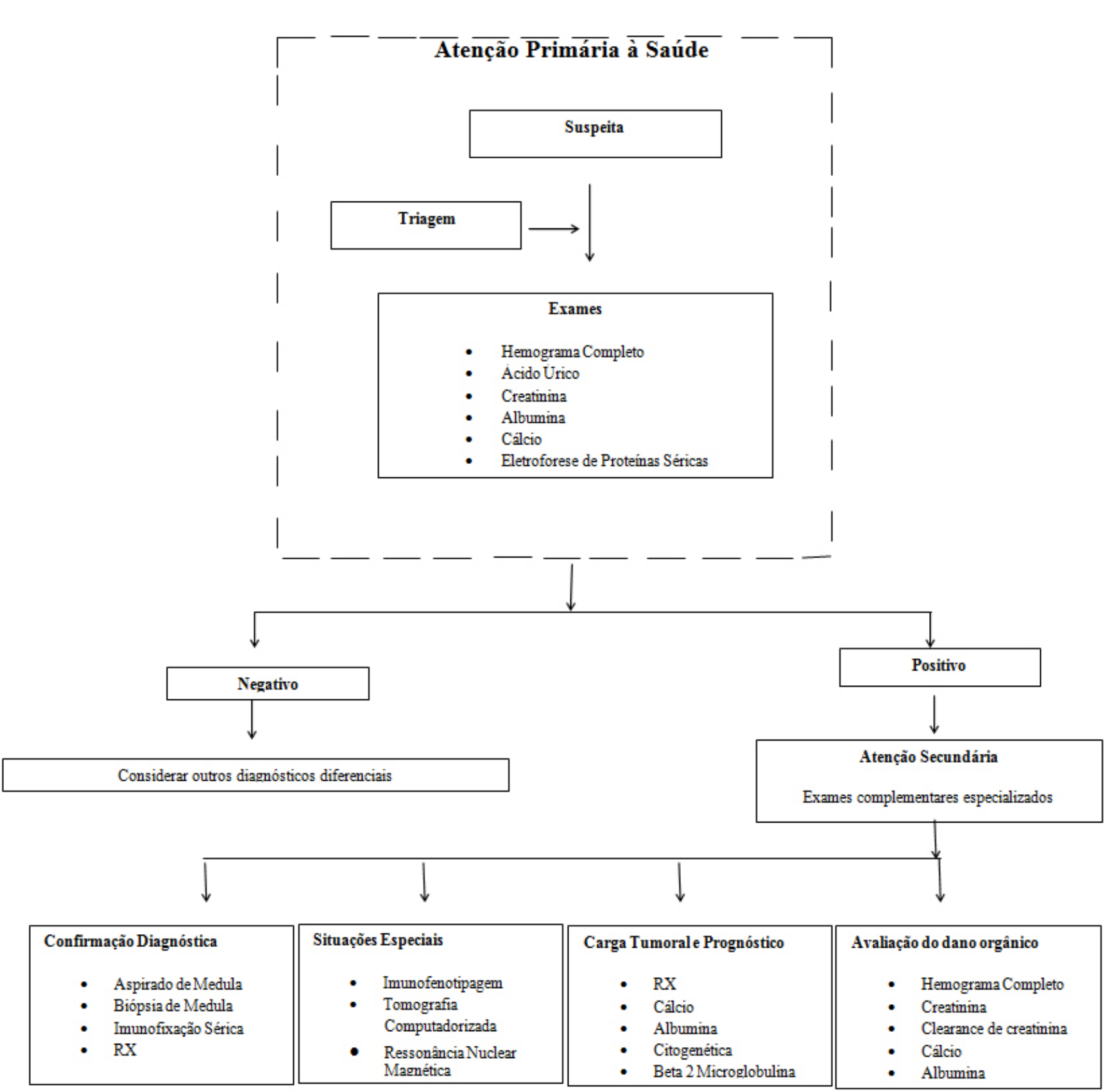

um paciente idoso, investiguem sua causa e considerem MM como diagnóstico diferencial. Mesmo sendo uma doença incurável, esse cuidado favorece o diagnóstico precoce, tratamento antecipado, maior sobrevida e qualidade de vida para pacientes com MM.

\section{Referências}

1. Aita MHC, Arantes LC, Aita BC, Comparação entre imunofixação e eletroforese na detecção precoce de recidivas do mieloma múltiplo. J.Bras. Patol.Med. Lab. [Internet]. 2015 Dec [acesso em 4 Jul 2018]; 51(6):359-36. Disponível: http://dx.doi.org/ 10.5935/16762444.20150057.

2. Silva ROP, Brandão KMA, Pinto PVM., Faria RMD, Clementino NCD, Silva CMF. et al. Mieloma múltiplo: características clínicas e laboratoriais ao diagnóstico e estudo prognóstico.Rev. Bras.Hematol.Hemoter. [Internet].2009 Abr[acesso em 4 Jul 2018];31(2): 63-68.Disponível: http://dx.doi.org/10.1590/S151684842009005000013.

3. Costa NA, Alves SM, Somenzi CC. Manifestações Clínicas que Auxiliam o Diagnóstico de Mieloma Múltiplo. Atas de Ciências da Saúde. [Internet]. 2014 Set[acesso em 4 Jul 2018]; 2 (4):113. Disponível: http://www.revistaseletronicas.fmu.br/index.php/ ACIS/article/view/592.

4. Rajkumar SV. Myeloma Today: Disease Definitions and Treatment Advances. Am J Hematol. [Internet]. 2016 Jan[acesso em 4 Jul 2018]; 91(1): 90-100. Disponível em: https://onlinelibrary. wiley.com/doi/epdf/10.1002/ajh.24236

5. Silva ROP, Faria RMD, Côrtes MJW, Clementino NCD, Faria JR, Moraes TEC. et al. Mieloma múltiplo: verificação do conhecimento da doença em médicos que atuam na atenção primária à saúde. Rev. Bras. Hematol. Hemoter. [Internet]. 2008 Dez [acesso em 
4 Jul 2018]; 30(6):437-444.Disponível: http://dx.doi.org/10.1590/ S1516-84842008000600004

6. Rajkumar SV. Updated diagnostic criteria and staging system for multiple Myeloma. Am Soc Clin Oncol Educ Book. [Internet]. 2016 Jan[acesso em 4 Jul 2018]; 35: 418-423. Disponível em: https:// www.ncbi.nlm.nih.gov/pubmed/27249749.

7. Hungria VTM, Crusoe EQ, Quero AA, et al. Mieloma Múltiplo. Associação Brasileira de Hematologia e Hemoterapia e Terapia Celular. Diretrizes. [Internet]. 2013 Out[acesso em 4 Jul 2018]; 31(2): 1-89.Disponível:http://amb.org.br/diretrizes/ Diretrizes/mieloma_multiplo/files /assets/common/downloads/ publication.pdf

8. Soares JPMR. Doença Óssea no Mieloma Múltiplo. [dissertação]. Porto: Universidade do Porto; 2013.

9. Harrison TR, Longo DL, Kasper D, Hauser S, Jameson J, Loscalzo J. Medicina Interna de Harrison. 19th ed. New York, NY: McGrawHill Medical; 2016. 2800 p712-716.

10. Fialho LFG, FulyPSC,Santos MLSC, et al. Validação de Diagnóstico dor Óssea e suas Intervenções de Enfermagem no Mieloma Multiplo. Cogitare Enferm. [Internet]. 2014 Out[acesso em 4 Jul 2018];19(4):755-60.Disponível:https://revistasufpr.brcogitare/ article/view/ 36683

11. Hungria VTM, Maiolino A, Almeida MSA, Crusoé EQ. Distúrbios dos Plasmócitose Doencas Correlatas. In: Zago MA, Paquini R, Falcao RP.Tratado de Hematologia - Fundamentos e Prática. Rio de Janeiro: Atheneu, 2001. p. 557-560.

12. Marta MJ, Falcão LM, Saavedra JA. Mieloma Múltiplo: a propósito de um caso raro de gamapatiabiclonalIgD/lambda -Abordagem diagnóstica e atitudes terapêuticas. Rev. Da Sociedade Portuguesa De Medicina Interna. [Internet]. 2009[acesso em 4 Jul 2018]; 9 (3): 1-21. Disponível em: https://www.spmi.pt/revista/ vol09/v09_n3_Mieloma_Multiplo.pdf.

13. Silva SMF. A Evolução da abordagem Terapêutica nos últimos 20 Anos. [dissertação]. Porto: Universidade do Porto; 2015.

14. PattrigH,Denise F, Pires EP, et all.Modelo assistencial em saúde: conceitos e desafios para a atenção básica brasileira.Rev.Ciênc.saúde coletiva. [Internet]. 2015 Jun[acesso em 4 Jul 2018];20 (6).

15. Ministério da Saúde (BR). Portarian ${ }^{\circ} 708$, de 06 de agosto 2015. Aprova as Diretrizes Diagnósticas e Terapêuticas do Mieloma Múltiplo; 2015.

16. Saccilotto IC. Análise de efetividade do Centro de Referência do Hospital de Clínicas de Porto Alegre para Tratamento de Mieloma Múltiplo. [Dissertação]. Porto Alegre: Universidade Federal do Rio Grande do Sul; 2014 